\title{
Non thermal effects of radiofrequency electromagnetic field exposure on neural cells
}

\author{
Rosaria Grasso ${ }^{1, *}$, Rosalia Pellitteri ${ }^{2}$, Santi Armando Caravella ${ }^{5}$, Francesco Musumeci ${ }^{1,4}$, Giuseppina Raciti $^{3}$, Agata \\ Scordino $^{1,4}$, Giovanni Sposito ${ }^{3}$, Antonio Triglia $^{1}$, and Agata Campisi ${ }^{3}$ \\ ${ }^{1}$ University of Catania, Department of Physics and Astronomy "Ettore Majorana”, 95123 Catania, Italy \\ ${ }^{2}$ Italian National Research Council, Institute for Biomedical Research and Innovation, 95126 Catania, Italy \\ ${ }^{3}$ University of Catania Department of Drug Sciences, Section of Biochemistry, 95123 Catania, Italy \\ ${ }^{4}$ National Institute for Nuclear Physics, Laboratori Nazionali del Sud, 95123 Catania, Italy \\ ${ }^{5}$ Temix Engineering s.r.1., 95039 Trecastagni, Italy
}

\begin{abstract}
The non-thermal mechanisms, underlying the damage induced on human cells by radiofrequency electromagnetic fields (RF-EMFs), are still unclear and only few studies reported about the effect of RFEMFs on self-renewal of neural progenitor cells. In this research, we investigated the influence of lowintensity RF-EMFs on Olfactory Ensheathing Cell (OEC) cultures, typical glia cells showing characteristics of stem cells. Cell cultures were exposed, in far-field condition, at $900 \mathrm{MHz}$ continuous and amplitude modulated EMFs for 10,15 and 20 min at $37^{\circ} \mathrm{C}$. The expression of OEC marker (S-100), stem cell marker (Nestin), cytoskeletal proteins (GFAP and Vimentin), apoptotic pathway activation by Caspase-3 cleavage and cell viability, were evaluated. Surprisingly $20 \mathrm{~min}$ of exposure to continuous or amplitude modulated $900 \mathrm{MHz}$ EMF induced a different and significant decrease in cell viability, some dynamic changes in the expression of the analysed markers and in the activation of the apoptotic pathway.
\end{abstract}

\section{Introduction}

Starting from the Industrial Revolution, the influence of the human being on his environment has gradually grown, introducing significant and perhaps irreversible changes. Just let consider the alterations of the climate that are ascribed to human activities such as the replacement of forests with cultivated areas, the intensive breeding and the increase in the concentration of greenhouse gases and aerosols in the atmosphere. However, the most drastic alteration of the natural conditions occurred with the introduction, in our vital environment, of a huge amount (compared to the preindustrial period) of electromagnetic fields characterized by a wide frequency range and by intensities some orders of magnitude greater than the natural background. An evident phenomenon in advanced societies is the fast increase in the use of mobile phones and the spread of wireless methodologies in the management of information through computers. These technologies are based on the use of so-called radio-frequency electromagnetic fields (RF-EMFs) having frequencies ranging from hundreds $\mathrm{KHz}$ to some $\mathrm{GHz}$. These fields, practically absent on the Earth's surface before the Industrial Revolution, today have permeated totally our living space.

Until a few decades ago it was believed that the nonionizing electromagnetic radiation was able to induce effects on the biological matter only through overheating of the tissues, inducing the so-called thermal effects.
This induced to define the concept of Specific Absorption Rate (SAR), that is the energy absorbed by tissue when exposed to RF-EMF. The SAR value is related to the properties of tissue (electrical conductivity, density), sample volume and the root mean square of the impinging electric field. The SAR definition includes the effect of electromagnetic filed frequency only indirectly, through the changes of tissue properties, but does not evaluate the possible effect of the electromagnetic field modulation. On this basis, the security protocols of various organizations, such as the WHO and ICNIRP, responsible for assessing the risks associated with the use of modern technologies, have been developed [1]. However, more recently, experimental evidence of the ability of RF-EMF to generate non-thermal biological effects at the cellular level is increasing [2-5] even if the heterogeneity of the RF-EMF, used for various technological applications, makes it difficult to find a cause-effect correlation.

In the paper, we present some results about our investigation on the non-thermal effect induced by a continuous RF-EMF at $900 \mathrm{MHz}(\mathrm{CW} 900 \mathrm{MHz})$ on a particular glial cell type, the Olfactory Ensheating Cells. The interest versus this cell line is increasing for several reason: an information transfer has been observed in the olfacto-hippocampal network [6]; it appears to be a promising tool for cellular therapy in spinal cord injury [7] and axonal growth [8]; it can stimulate axonal regeneration and functional restoration in the lesion of

Corresponding author: rosaria.grasso@ct.infn.it 
Nervous System [9]. To better understand the effect induced by modulation, we exposed cell cultures also to an amplitude modulated $900 \mathrm{MHz}$ ( $\mathrm{AM} 900 \mathrm{MHz})$. Moreover we evaluated the effect of exposure time (10, 15 and 20 minutes).

\section{Materials and methods}

\subsection{Materials}

Normal Goat Serum, $200 \mathrm{mM}$ L-glutamine, collagenase Penicillin-Streptomycin solution, heat inactivated Foetal Bovine Serum Dulbecco's Modified Eagles Medium, $0.05 \%$ trypsin- $0.02 \%$ EDTA solution, were purchased by Invitrogen (ThermoScientific, Milan, Italy). Monoclonal mouse anti Vimentin clone V9 (cod. 2012-03) and polyclonal rabbit anti GFAP (cod. 201502) were from DAKO. 1-(4,5-Dimethylthiazol-2-yl)-3,5diphenylformazan (MTT), were from Sigma-Aldrich (Milan, Italy).

\subsection{Animals and OEC cultures}

The mouse pups were provided by Envigo RMS s.r.l. Italy (stock: C57BL6J). Animals were kept in a controlled environment $\left(23 \pm 1^{\circ} \mathrm{C}, 50 \pm 5 \%\right.$ humidity $)$ with a $12 \mathrm{~h}$ light/dark cycle with food and water available ad libitum. All the experimental procedures were carried out according to the Italian Guidelines for Animal Care (D.L. 116/92 and 26/2014), which are in compliance with the European Communities Council Directives (2010/63/EU) and were approved by the Ethical Committee at the University of Catania (Catania, Italy).

Primary OECs were isolated from mouse P2 olfactory bulbs and purified as previous described [10].

\subsection{RF-EMF exposure}

The RF- EMF was emitted by a double horn antenna (ETS-Emco- 3115) plugged to an Agilent-8648D signal generator. The amplitude modulation of sinusoidal waveform was obtained by an external oscillator (Philips-PM5127). Two different irradiation conditions at the same amplitude around $6 \mathrm{~V} / \mathrm{m}$ were used: continuous radiofrequency at $900 \mathrm{MHz}$ wave (CW $900 \mathrm{MHz}$ ) and radiofrequency at $900 \mathrm{MHz}$ wave modulated in amplitude with a frequency of $50 \mathrm{~Hz}$ and modulation index 0.27 (AM $900 \mathrm{MHz}$ ) for 5, 10, and 20 min of exposure time. Irradiation was performed in farfield condition, ensuring a good uniformity of the electromagnetic field at the sample.

During the exposure, the cell cultures were maintained in a thermal bath at $37^{\circ} \mathrm{C}$.

\subsection{MTT assay and immunocytochemical procedure}

OECs cell viability were performed on 96 multiwell flat bottomed $200 \mu \mathrm{l}$ microplates $\left(1 \times 10^{4}\right.$ cells $/$ well $)$ as described in ref. 11. The expression of cytoskeleton proteins were performed on coverslips $\left(1 \times 10^{4}\right.$ cells/well) as reported in ref. 12 .

Data were statistically analysed using One-Way analysis of variance (ANOVA) followed by a post hoc Holm-Sidak test to estimate significant differences among groups. Data were reported as mean \pm SD of four separated experiments in duplicate, and differences between groups were considered to be significant at $\mathrm{p}<$ $0.05, \mathrm{p}<0.01, \mathrm{p}<0.001$.

\section{Results and discussion}

This study represents the first step of a more complex one, involving a long and deep investigation. We assessed the effect of electromagnetic field exposure on the main cytoskeletal components which are more relevant in terms of OEC physiology. We analysed cytoskeleton protein expression, as it represents the first signal of cellular alteration. It is well known that the cytoskeleton plays a key role in the cell architecture and it is responsible for intracellular trafficking of endogenous and exogenous molecules and stimuli.

We investigated cell viability using MTT assay and, through immunocytochemical procedures, the expression of Glial Fibrillary Acidic Protein (GFAP), Vimentin and Nestin, that are proteins implicated in the formation of Intermediate Filaments, as well as S100 protein, OEC marker, and the activation of apoptotic pathway by analysing caspase-3 cleavage.

For the experiments, cells were divided in four groups: control cells maintained into the incubator at $37^{\circ} \mathrm{C}$ in ambient of humidified air and $\mathrm{CO}_{2}(95 \%-5 \%)$, to verify the normal cellular status (Ctrl); cells maintained in water thermal baths at $37^{\circ} \mathrm{C}$ for 10,15 and 20 minutes (Sham); cells maintained in water thermal baths, exposed to $\mathrm{CW} 900 \mathrm{MHz}$ for 10,15 and 20 minutes (CW 900 $\mathrm{MHz}$ ); cells maintained in water thermal baths, exposed to AM $900 \mathrm{MHz}$ for 10, 15 and 20 minutes (AM 900 $\mathrm{MHz})$.

Here we will present, schematically, only the results concerning cell viability, GFAP and Vimentin.

The MTT assay showed no significant change in cell viability in sham versus control cells at 10,15 and 20 minutes exposure time. The continuous and, in particular, amplitude modulated $900 \mathrm{MHz}$ were able to induce a significant decrease in cell viability respect to respective shams only when the exposure was for 20 minutes.

For what concern immunocytochemistry analysis, the exposure to RF-EMFs for 10 minutes did not induce effect on cell cultures. Therefore, we report about the quantitative analysis of GFAP (fig. 1) and Vimentin (fig. 2) expression levels in OECs un-exposed and exposed to EMFs for 15 and 20 minutes.

The control cell showed a low number of GFAP positive cells (fig. 1). The absence of $\mathrm{CO}_{2}$ (Sham) for $20 \mathrm{~min}$ induced a light, even if significant, increase in the GFAP positivity cell. The exposure to $\mathrm{CW} 900 \mathrm{MHz}$, both for $15 \mathrm{~min}$ and $20 \mathrm{~min}$, induced a highly significant increase in GFAP expression level. Instead, the exposure to AM 
$900 \mathrm{MHz}$, both for $15 \mathrm{~min}$ and $20 \mathrm{~min}$, induced a decrease in GFAP expression level respect to cell cultures exposed to $\mathrm{CW} 900 \mathrm{MHz}$, and, at $20 \mathrm{~min}$, also respect to sham.

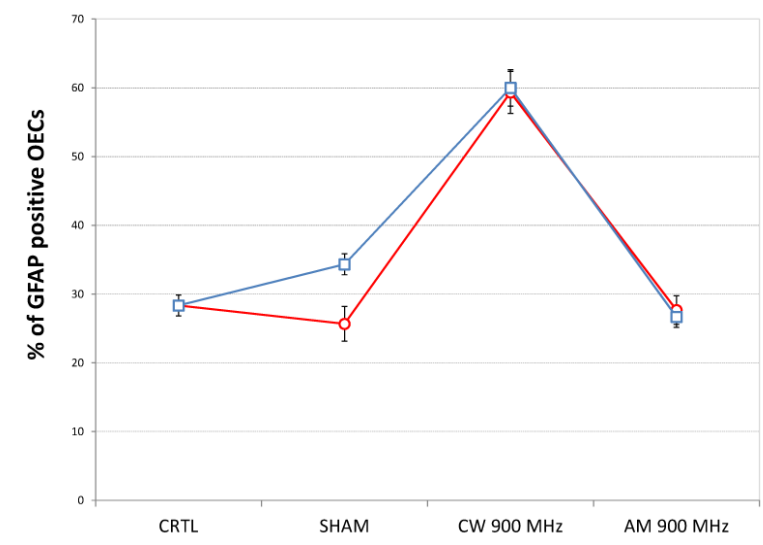

Fig. 1. $\%$ of GFAP positive OECs on control, sham, CW 900 MHz, AM $900 \mathrm{MHz}$ after exposure time of (०) $15 \mathrm{~min}$, () 20 $\mathrm{min}$. Values of four separated experiments are reported as mean $\pm \mathrm{SD}$.

Both control and sham showed a low positivity for Vimentin (fig. 2). The exposure to continuous $900 \mathrm{MHz}$ induces an high significant increase in Vimentin positive OECs. A recovery effect seems to be activated after 15 min exposure time. The AM $900 \mathrm{MHz}$ induced a highly significant increase of the Vimentin positive cells number at $15 \mathrm{~min}$ exposure time and its drastic decrease at $20 \mathrm{~min}$ not only respect to the cell cultures exposed to CW $900 \mathrm{MHz}$ but also respect to control and sham.

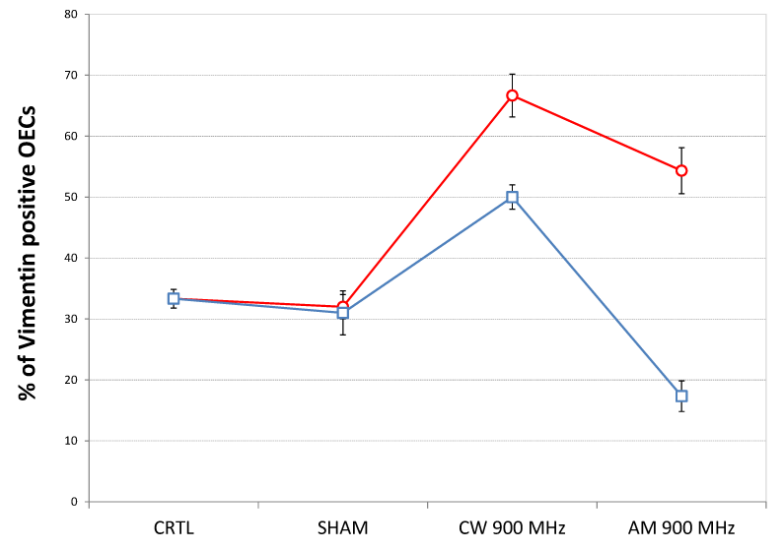

Fig. 2. \% of Vimentin positive OECs in control, sham, CW 900 $\mathrm{MHz}, \mathrm{AM} 900 \mathrm{MHz}$ after exposure time of (०) $15 \mathrm{~min},(\square) 20$ min. Values of four separated experiments are reported as mean $\pm \mathrm{SD}$.

In conclusion, these results, together to others not showed here, highlighted that continuous or amplitude modulated $900 \mathrm{MHz}$ exposure was able to differently alter the cytoskeletal proteins at various levels and that these effects were function of exposure time. In particular, the results on GPAF expression levels indicated that the exposure to $\mathrm{CW} 900 \mathrm{MHz}$ enhances the OECs ability to differentiate versus astroglial cell type, while AM $900 \mathrm{MHz}$ exposure for 20 min induces a decrease respect to the Sham. Moreover, the experimental results on Vimentin expression levels pointed out that the exposure to $\mathrm{CW} 900 \mathrm{MHz}$ and $\mathrm{AM}$ $900 \mathrm{MHz}$ can induce different effect on the cytoskeleton remodelling. It is worth to note that the latter one, highlighted through Vimentin, activates signalling pathways having got a role both as cell viability modulator and cell structure constituents.

\section{References}

1. High Frequency review. ISBN 978-3-934994-10-2 (2009)

2. S.Cucurachi, W.L Tamis, M.G. Vijver, W.J. Peijnenburg, J.F. Bolte, G.R. de Snoo. Environ. Int. 51, 116 (2013)

3. A. Campisi, M. Gulino, R. Acquaviva, P. Bellia, G. Raciti, R. Grasso, F. Musumeci, A. Vanella, A. Triglia, Neurosci. Lett. 473, 52 (2010).

4. C. Chen, Q. Ma, C.Liu, P. Deng, G., Zhu, L. Zhang, M. He, Y. Lu, W. Duan, L. Pei, et al., Sci. Rep. 4, 5103 (2014).

5. M. Eghlidospour, A. Ghanbari, S.M.J. Mortazavi, H. Azari, Anat. Cell. Biol. 50, 115 (2017).

6. B. Gourévitch, L.M. Kay, C. Martin, J. Neurophysiol. 103, 2633 (2010).

7. R.M. Gómez, M.Y. Sánchez, M. Portela-Lomba, K. Ghotme, G.E. Barreto, J. Sierra, J., M.T. MorenoFlores, Glia. 66, 1267 (2018).

8. F. Chehrehasa, L.C. Windus, J.A. Ekberg, S.E. Scott, D. Amaya, A. Mackay-Sim et al., Mol. Cell. Neurosci. 45, 277 (2010).

9. S. C. Barnett, J.S. Riddell, J. Anat. 204, 57 (2004)

10. R. Pellitteri, M. Spatuzza, A. Russo, S. Stanzani, Neurosci. Lett. 417, 24 (2007).

11. A. Campisi, M. Spatuzza, A. Russo, G. Raciti, A. Vanella, S. Stanzani, R. Pellitteri, Neurosci. Res. 72, 289 (2012).

12. R. Pellitteri, R. Bonfanti, M. Spatuzza, M.T. Cambria, M. Ferrara, G. Raciti, A. Campisi, Mol. Neurobiol. 54, 6785 (2017). 